\title{
Claudel, l'Histoire et la Diplomatie
}

\author{
Eric Touya de Marenne \\ Clemson University
}

Dans « La crise de l'esprit », Paul Valéry affirmait après la tragédie de la première guerre mondiale que « nous autres, civilisations, nous savons maintenant que nous sommes mortelles. " Au-delà du drame humain, c'est le sort de l'humanité qui se jouait par le biais d'une mise en question profonde des fondements de notre civilisation et des moyens par lesquels nous pouvions les sauvegarder :

\begin{abstract}
Tant d'horreurs n'auraient pas été possibles sans tant de vertus. Il a fallu, sans doute, beaucoup de science pour tuer tant d'hommes, dissiper tant de biens, anéantir tant de villes en si peu de temps; mais il a fallu non moins de qualités morales. Savoir et Devoir, vous êtes donc suspects ${ }^{1}$ ?
\end{abstract}

Dans le monde actuel qui est le nôtre, confronté à la crise économique, au dialogue incertain entre les peuples, les cultures et les religions, et à la menace de la guerre et du terrorisme, la réflexion de Paul Valéry n'a rien perdu de son sens et de son importance. Représentant de la France en Chine au Japon puis aux Etats-Unis, parmi d'autre pays, Paul Claudel n'était pas lui non plus étranger à cette crise d'un intellect faisant face désormais par sa puissance même à sa propre auto destruction, de même qu'il reconnaissait dans le progrès de la technologie et de la machine l'anéantissement possible de l'humanité. La catastrophe de la deuxième guerre et son aboutissement à Hiroshima et Nagasaki ne devaient pas lui donner tort. On ne saurait sous-estimer à cet égard le rôle qu'a joué l'ambassadeur Paul Claudel aux Etats-Unis, à l'aube d'une ère dans 


\section{Eric Touya de Marenne}

laquelle nous sommes rentrés (celle du XX siècle) et dont il est possible que nous ne puissions jamais ressortir. Nous chercherons donc à tirer quelques enseignements de la réflexion claudélienne sur un sujet encore et toujours contemporain.

La première leçon est que la diplomatie est un art et un métier qui s'acquiert, la guerre résultant le plus souvent de son absence et de notre méconnaissance. C'est le 22 juillet 1941, dans les plus mauvais jours, qu'il en fait l'observation dans «Quelques réflexions sur le métier diplomatique » : «Pourquoi aucun d'eux [il s'agit de ses prédécesseurs] n'avait songé à nous parler en technicien du métier diplomatique. Que de conseils utiles n'auraient-ils su nous donner sur le noble art de conduire vis-à-vis de l'étranger les affaires du pays! » Il soulignait l'ignorance de l'autre qui prévalait même parmi les spécialistes du Quai d'Orsay :

Une première remarque qui paraîtra d'abord paradoxale, c'est que le ministère des Affaires étrangères ne connaît pas l'étranger [...] Bien entendu, je ne nie pas que [les agents du Service central] aient de l'étranger cette connaissance superficielle que l'on peut acquérir par la lecture, celle des lettres et télégrammes qui arrivent chaque jour, celle beaucoup plus superficielle des journaux, et celle bien exceptionnelle je le crains des livres et des revues qui essayent de nous donner une physionomie étudiée, réfléchie et synthétique, des parties et des moments du scénario international.

La technologie incarnée par le progrès de l'aéronautique ne pouvait à elle seule réduire à néant notre incapacité à comprendre l'étranger et à résoudre le paradoxe de la distance géographique et de la distance morale. Claudel l'explique dans " L'Avion et la diplomatie » rédigé en 1936, année durant laquelle se confirme la montée des fascismes et des nationalismes :

Il la survole. Entre les nations il raccourcit la distance physique, mais que penser de la distance morale? La radio 
elle-même, plus immédiate encore, qui utilise un élément plus subtil et plus spirituel que l'air même, et qui en quelques tours de bouton nous abouche à toutes les voix de l'univers, elle a démesurément élargi la sphère de notre intérêt, est-il sûr qu'elle ait dilaté celle de nos sympathies, ou même celle de notre intelligence? A cette question, le nationalisme exaspéré qui partout a coïncidé avec le développement et l'accélération des moyens de communication, se charge de fournir une réponse. On dirait que les hommes, plus ils se connaissent et moins ils s'aiment. Plus ils se touchent et plus ils se rétractent, plus ils prennent une conscience exclusive d'eux-mêmes, plus ils s'attachent à leur caractère propre et à leurs différences fondamentales. Il n'y a pas d'invitation extérieure qui ne provoque une réaction dans un organisme non préparé. [...] Telles sont les conditions psychologiques parmi lesquelles le diplomate, aujourd'hui, a à exercer sa petite industrie. [...] Ainsi l'avion qui s'était déjà fait tant bien que mal notre professeur de géographie s'est constitué notre répétiteur de droit international ${ }^{2}$.

Quatre textes sur les Etats-Unis mettent en question et offrent une réponse aux problématiques soulignées jusqu'ici : 1 . les fins de l'intelligence de l'humanité qui touchent à la question même de sa survie; 2 . la méconnaissance profonde de l'autre qui empêche avec lui toute réconciliation possible; 3 . les frontières qui séparent le progrès technologique du progrès moral; 4 . le rôle du diplomate face à ces défis insurmontables.

Dans «L'Élasticité américaine » rédigé en cette même année 1936, Claudel prend la mesure d'un peuple étranger qui par son caractère, sa capacité à rebondir, par son ouverture d'esprit et sa générosité sera en mesure quelques années plus tard de vaincre les totalitarismes au nom de la liberté :

Il y a dans le tempérament américain une qualité que l'on traduit là-bas par le mot resiliency pour lequel je ne trouve 


\section{Eric Touya de Marenne}

pas en français de correspondant exact, car il unit les idées d'élasticité, de ressort, de ressource et de bonne humeur. Une jeune fille perd sa fortune, elle se mettra sans grogner à laver la vaisselle et à fabriquer des chapeaux. Un étudiant ne se croira pas déshonoré de travailler quelques heures par jour dans un garage ou dans un café. J'ai vu l'Amérique à la fin du président Hoover, à l'une des heures les plus tragiques de son histoire, quand toutes les banques avaient fermé et que la vie économique était suspendue. L'angoisse étreignait les cœurs, mais la gaieté et la confiance éclairaient tous les visages. A entendre les propos échangés, on aurait dit qu'il s'agissait d'une énorme plaisanterie. Et si quelques financiers se jetaient par la fenêtre, je ne puis m'empêcher de croire que c'était dans l'espérance fallacieuse de rebondir.

[...] L'expérience n'a pas ici le même sens qu'en Europe. Il ne s'agit pas d'une sagesse procurée par le passé, mais d'une aventure avec l'avenir, d'un engagement tout neuf avec un partenaire dont les réalisations ont toujours surpassé les promesses. L'Américain a besoin d'horizons et il a besoin de camarades autour de lui. Et alors en avant! de nouveau, il se met à entendre le fifre et la grosse caisse et son jarret à marquer la cadence! C'est cette pulsation rythmique et nerveuse pareille aux pistons d'une machine à vapeur entrecoupant le ronflement cyclique de la dynamo qui se fait sentir à travers toute la vie américaine et dont le jazz est la suprême expression ${ }^{3}$.

Dans "L'Amérique et nous », également écrit pendant cette année déterminante de 1936, le diplomate retraité depuis deux ans s'attache à définir les liens qui unissent deux nations par un passé qu'ils partagent, les dangers auxquels ils sont confrontés, et l'avenir qu'ils souhaitent entrevoir animés d'un même esprit :

Quand j'arrivai en Amérique au printemps de 1927, je trouvai un pays encore vibrant du souvenir des épreuves 
et des victoires de la Grande Guerre. Les noms de Foch, de Joffre, de Pétain, de Gouraud, de Clémenceau se mêlaient dans la légende et dans la ferveur populaire et rejoignaient sur les autels de la tradition ceux de La Fayette, de Rochambeau, et de Grasse. La gloire de Saint-Mihiel et de l'Argonne égalait celle de Yorktown et de Gettysburg. Mon premier devoir fut de présider dans les grandes villes de l'Union d'immenses réunions de vétérans où la Marseillaise était chantée et la France évoquée avec un enthousiasme et une sincérité qui réjouissaient le cœur. Chaque poste de vétérans sur cet immense territoire était comme un foyer où ce sentiment d'amitié était entretenu et propagét.

C'est dans ce même texte que Claudel évoque la question épineuse de la dette de la France contractée pendant la première guerre. Ce passage nous permet d'entrevoir les difficultés auxquelles s'expose le diplomate quand ses vues sont en contradiction avec les positions officielles de son gouvernement. Il montre aussi combien l'Histoire donnera raison à Claudel et que les mêmes choix et évènements réapparaissent au fil de l'Histoire :

Une nouvelle chance nous fut offerte. L'administration républicaine venait d'être balayée par l'avalanche et Franklin Roosevelt avait été nommé à la Présidence. Presque aussi tôt, j'eus avec ce grand homme d'État, qui partage seul dans mon esprit l'admiration que j'ai vouée au roi Albert, deux entrevues qui m'ont laissé le plus émouvant souvenir. [...] La conversation s'engagea aussitôt. J'en ai feuilleté les minutes. Elle s'étendit à toutes les questions alors pendantes dans le monde et témoigna sur presque tous les sujets d'une communauté de vues. Elle se résuma sur la question des dettes à un seul appel : « Je vous en prie, aidez-moi, faites quelque chose. Vous voyez ce que j'ai déjà accompli en ces quelques semaines de Présidence. Aujourd'hui, je vous tends la main, 
Mr. Herriot, avec la même loyauté que Wilson le fit jadis à Joffre et à Viviani. (Textuel). Faites un geste aussi restreint que vous le voudrez et vous pourrez compter que je mettrai au service d'un accord toute l'autorité dont je dispose en ce moment auprès du Congrès et qui, vous ave $z$ pu vous en rendre compte, n'est pas médiocre. » Il ne s'agissait pas de mots en l'air. Il y avait en ce moment dans le tiroir du Président un plan extrêmement ingénieux, rédigé par un technicien de son entourage, et qui aurait consacré une diminution considérable de nos obligations.

"Ce plan ne fut même pas examiné sérieusement à Paris. L'opinion française était butée. Elle envisageait non seulement sans regrets, mais avec une espèce d'allégresse cette rupture déchirante et peut-être définitive avec nos amis d'Amérique qui, à moi, après tant de peine d'espérance et d'efforts, causait un véritable désespoir. Trois ans se sont maintenant écoulés et nous pouvons faire le bilan de l'opération à laquelle nous nous sommes prêtés de gaité de cœur. Je le demande à tous les lecteurs de bonne foi : abstraction faite de tout ce que nous avons perdu de l'autre côté de l'Atlantique et qui est démesuré, avons-nous à nous louer en Europe et en France de nous être aliéné l'Amérique? Avons-nous eu raison de repousser la main puissante et sage que le grand Président nous tendait ${ }^{5}$ »

Trois ans plus tard, les évènements donnaient donc raison à notre auteur. Rédigé en janvier 1939, « Devant le vertige européen » témoignait de l'inexorable suprématie de la guerre sur les efforts d'une paix improbable que la Société de Nations et le plan Briand-Kellogg avaient courageusement cherché à soutenir :

J'éprouve le même sentiment à la lecture de la littérature totalitaire. C'est la même inspiration d'en bas, mais cette fois sans la moindre phosphorescence de génie ou de 
talent. Des hurlements de loup, des jappements de roquet enragé, un mépris atroce de toutes ces valeurs, de toutes ces vertus qui ont toujours fait la parure de l'humanité et le ciment de la civilisation, et sur tous ces visages à la fois obscurs et flamboyants d'une passion démoniaque, l'expression hideuse de ce sentiment qui défigure davantage, celle de l'envie et celle de la haine. Plus de vérité, plus de religion, plus d'amour, plus de droit, plus de justice, plus de morale. C'est la guerre des classes et c'est la guerre des races. [...] Il s'agit de construire sur la base de doctrines aussi enfantines et aussi stupides que le marxisme et le racisme, on s'aperçoit que la passion ne suffit pas. Que penser de ce mot d'ordre de totalité qui commence par mutiler l'individu et par lui boucher l'âme, qui déclare la guerre, comme on le fait en Allemagne, à toute variété d'opinions, à la fois aux Juifs, aux catholiques, aux protestants et aux monarchistes, aux artistes, à tout ce qui essaie une pensée indépendante ${ }^{6}$.

Dans un autre texte rédigé en avril 1945 et intitulé « Mes souvenirs sur Franklin Roosevelt ", Claudel rendait hommage au président américain dont la magnitude était à la hauteur du courage et de la persévérance de son peuple :

Bien que Roosevelt ne dût occuper officiellement son poste qu'au mois de février, et nous étions en novembre, je me résolus à lui demander par des relations communes une entrevue amicale, qu'il m'accorda aussitôt. [...] Ce fut le point de départ de longues et importantes conversations qui continuèrent à Washington et se prolongèrent jusqu'au moment de mon départ d'Amérique en mars 1933. Elles me permirent d'apprécier la justesse d'une parole que j'ai entendue souvent dans la bouche de mes amis américains, c'est qu'on en apprend plus sur le caractère d'un homme en une heure 


\section{Eric Touya de Marenne}

de discussions d'affaires, qu'en des années de relations mondaines. L'homme que j'avais devant moi [...] cette figure ouverte qui respirait assez de confiance en soimême pour ne pas se refuser l'arme et l'attrait de la sincérité, le journal du matin avait bien raison de dire que tout cela appartenait à la catégorie $A$ first. Il se levait. On entendait un bruit de ferraille et le rebord d'une armature d'acier apparaissait sous le pantalon. Ce géant était un infirme. Un destin cruel l'avait fauché par la base.

[...] Roosevelt appartenait à un type humain d'une toute autre envergure. [...] Chez lui, une union magnifique de deux tempéraments. L'un, celui qu'il tenait de la nature : magnanimité, audace, allant jusqu'à la témérité, promptitude, courage, obstination (à laquelle il faut ajouter une certaine difficulté au pardon). L'autre, résultant à la fois de la maladie et de cette rude éducation de l'homme politique dont la vie se passe à donner et à recevoir des coups. L'habileté et l'expérience d'un lutteur, en même temps que le calcul et la patience d'un infirme à qui les circonstances permettent la méditation?

Claudel avait été non seulement le témoin mais aussi un des acteurs de l'Histoire. Dans le dernier texte que je citerai aujourd'hui « Adieu à l'Amérique ", l'ambassadeur est fidèle à ses convictions chrétiennes. Il nous fait part, dans un discours prononcé le 10 avril 1933, de ce qui est pour lui le but ultime du diplomate : celui de la recherche de la paix entre les hommes :

Vous trouverez naturel qu'au moment où je vais quitter l'Amérique, je vous convie à envisager avec moi le chemin que nous avons parcouru ensemble et à apprécier l'œuvre accomplie. Celle qui me laisse le plus de satisfaction est la part que j'ai eu à prendre en qualité de négociateur à ce grand effort de pacification internationale qu'on appelle le pacte Briand-Kellogg ou Pacte de Paris, et qui un an plus tard recevait la signature de 
tous les gouvernements de la planète à l'exception de deux ou trois.

[...] Bien entendu les signataires et les partisans du pacte de Paris ne pouvaient s'attendre qu'il suffirait d'un simple document, d'une promesse écrite, si solennelle qu'elle fût, pour changer la nature humaine, pour brider instantanément les passions les plus violentes du cœur humain. [...] Mais personne ne saurait nier qu'aux trop faciles et néfastes solutions de la guerre, il existe aujourd'hui un obstacle et une alternative. L'opinion du monde, la conscience du monde, est désormais pourvue d'un moyen d'expression, d'un organe qui donne forme à son sentiment de solidarité à l'égard de tout acte qui constitue pour un intérêt particulier une infraction au droit de tous. Un arbre a été planté dont la tempête peut tourmenter les branches, mais dont les racines sont saines et solides ${ }^{8}$.

Que pouvons-nous retenir aujourd'hui de l'enseignement de Claudel témoin et acteur de l'Histoire, nous qui sommes aujourd'hui attentifs à son déroulement et aspirons à un meilleur avenir? Tout d'abord que ce qui prime dans la diplomatie n'est pas la raison d'État chère au machiavélisme par laquelle la guerre devient le prolongement nécessaire des relations internationales. Notre auteur y voit plutôt un art et un devoir : celui de privilégier les solutions pacifiques, de représenter son pays et d'agir en son nom, si tant est que celui-ci demeure à l'écoute de cette musique que le monde empêche d'entendre.

\section{Notes}

1 Paul Valéry, CEurres I, édition établie et annotée par Jean Hytier. Paris : Editions Gallimard, 1957.

2 Paul Claude, "L'Aviation et la diplomatie ", Euvres en prose, préface de Gaëtan Picon, textes établis et annotés par Jacques Petit et Charles Galpérine. Paris : Editions Gallimard, 1965, p. 1297-1301. 


\section{Eric Touya de Marenne}

3 "L'élasticité américaine », Euvres en prose, p. 1204-1205.

4 «L'Amérique et nous », Euvres en prose, p. 1209.

5 Ibid., p. 1212-1213.

6 « Devant le vertige européen », CEuvres en prose, p. 1319-1320.

7 «Mes souvenirs sur Franklin Roosevelt ", Euvres en prose, p. 12141216.

8 « Adieu à l'Amérique », Euvres en prose, p. 1218-1219. 